\title{
Two-Color Coherent Photodissociation of Nitrogen Oxide in Intense Laser Fields
}

\author{
Julian Juhi-Lian Ting \\ Physics Institute, Ching-hua University, \\ Hsin-Chu, Taiwan 30043, Republic of China *
}

(November 10, 2018)

\begin{abstract}
A simple one-dimensional semi-classical model with a Morse potential is used to investigate the possibility of twocolor infrared multi-photon dissociation of vibrationally excited nitrogen oxide. The amplitude ratio effects and adiabatic effects are investigated. Some initial states are found to have thresholds smaller than expected from single-mode considerations and multiple thresholds exist for initial states up to 32 .
\end{abstract}

PACS: $42.50 . \mathrm{Hz}$

Two-color lasers interacting with molecules with a phase difference might alter greatly the behavior of molecular dissociation. According to the calculation by Charron et al for $\mathrm{H}_{2}^{+}$with varied phase shifts between two laser fields, [1] the above-threshold dissociation of $\mathrm{H}_{2}^{+}$can be strongly enhanced by combining the laser radiation with a harmonic and varying the relative phase between the two radiations. However their calculation is for only the first excited state.

It has become possible to transfer population of nitrogen oxide (NO) efficiently to higher states: Yang et al reported experiments in which molecules were populated into an initial vibrational state as great as $n=25$ by stimulated-emission pumping (SEP) [2 whereas Schiemann et al showed how to populate to a state $n=6$ by a stimulated Raman process involving adiabatic passage (STIRAP) using pulsed lasers [3]. Therefore it is desirable to know about the behavior of a diatomic molecular at other excited states under intense two-color laser fields.

In this work, previous results [4 for single-mode lasers using a Morse potential were extended for a laser with its own third harmonic. Instead of considering $\mathrm{H}_{2}^{+}$, as did by Charron et al, we considered NO, which can serve to answer the question whether the observed behavior is universal for all diatomic molecules. The main purpose of this work was to find the dissociation thresholds of NO with two-color lasers; guided by results of two-color lasers we returned to find lower thresholds under single-mode.

The model used is simple and cannot distinguish between above-threshold dissociation and other effects such as stimulated emission, multiphoton absorption, bond softening and suppression of dissociation as did Charron et al. However, we can assess which effect has the most significant influence. As above-threshold dissociation is the most important contribution for photodissociation of molecules, according to Charron et al, one would expect our results to be consistent with theirs.

We consider an isolated non-rotating NO molecule interacting with plane-polarized harmonic laser fields. The dimensionless Hamiltonian of a free diatomic molecule as a Morse oscillator is

$$
H_{0}=\frac{p^{2}}{2}+\frac{\left(1-e^{-z}\right)^{2}}{2}
$$

in which $z$ and $p$ are the dimensionless coordinate and the corresponding momentum respectively. The parameters of NO are given in Ref. 幽.

The effects of neglecting the rotational contribution are two fold. Firstly, additional selection rules might apply if one considered the rotational contribution. Secondly, angular momentum contributes an effective potential $l(l+1) /\left(z+r_{e}\right)^{2}$, with $r_{e}$ the equilibrium inter-nuclei distance, which alters the asymptotic behavior of the effective potential of the radial Schrödinger equation at large separation. [5] The Hamiltonian without the angular part corresponds to a model of DNA denaturation in the continuum limit. [6]

We concluded in previous work that an exponential form of coupling is suitable for molecular calculation. With two color-lasers the interacting Hamiltonian reads

$$
\frac{A_{0} \Omega}{2}(z+a) e^{-(z+a) / b}\left(A_{1} \sin (\Omega t)+A_{2} \sin (3 \Omega t+\phi)\right) \sin ^{2}\left(\frac{2 \pi t}{T}\right),
$$

in which $\Omega$ and $A_{j}, j=0,1,2$, are dimensionless frequency and amplitudes respectively. The variables $a$ and $b$ were chosen to have the values 2 and 1 respectively in the calculation. The definition of the dissociation rate is [7]

$$
P_{\text {diss }}(t)=1-<\psi_{I}(t) \mid \psi_{I}(t)>
$$

in which $\mid \psi_{I}(t)>$ is the interacting wavepacket. We take $T$, the pulse duration, to be 300 (or 600) optical cycles. With this choice the pulse duration lies in the nanosecond range. The dimensionless amplitude $A=1$ corresponds to a laser intensity $320 \mathrm{TW} / \mathrm{cm}^{2}$.

The numerical method used is a fast Fouriertransformed grid method. [8] All calculations were done dimensionlessly at $\Omega=0.9$, which is slightly less than the transition frequency between the ground state and the first excited state of the free Morse oscillator. Each dissociation threshold curve was obtained from more than 1000 points of calculations. There is no general rule to find out a second or a third transition for each initial state. However, it is reasonable to expect that neighboring states, or other similar states, should have similar thresholds. 
Firstly, the previous result of a single-mode laser is reproduced in fig.1, with several additional lower thresholds found and $n_{0}=1$ is found to have three thresholds.

Secondly, for two-color lasers, four curves for $A_{1}=1$ and $A_{2}=\sqrt{3}$ with $\phi=0, \frac{\pi}{2}, \pi, \frac{3 \pi}{2}$ in Eq. (2) are plotted in fig.2. As before we computed both $T=300$ and $T=600$. For over half the initial states a phase difference $\pi$ reduce the value of $A_{c}$ most effectively. In order to compare the effect of the phase difference, we replotted fig. 2 in fig. 3 for $T=300$ and 600 respectively. Although we simulated with more than twice the laser power in the present case than in the single-mode case, the threshold amplitudes were not diminished correspondingly, contrary to results of Charron et al. By comparing $A_{c}$ for $T=300$ and for $T=600$ one obtains hints about whether a particular value of $n_{0}$ is dissociated by itself or by transition to other states. According to perturbation theories [9] the shorter is the light pulse, the more stationary states are excited and vice versa. The fact that a phase difference $\pi$ does not invariably result in the smallest dissociation threshold contrasts with our intuition that zero phase difference has the largest intensity, but is consistent with the calculation on first excited states by Charron et al. However, the difference diminished as $T$ became larger, i.e. fig. 3(b). Multiple thresholds were found up to an initial state equal to 32 for a phase difference $\pi$.

Thirdly, a modified amplitude ratio for $A_{1}=1$ and $A_{2}=2$ was also investigated; the results are plotted in fig. 4 with $T=300$, comparison with fig.3(a). At a phase difference $\pi / 2, n_{0}=4$ has four thresholds. A phase difference $\pi$ still has the most lowest thresholds whereas a phase difference $\pi / 2$ has no lowest threshold.

There are some inherent limitations to this model. One suspects that multiple thresholds at higher initial states can also be found with calculations of finer amplitude resolution. Secondly, $n_{0}>44$ shows almost the same $A_{c}$ in all calculations, which is also attributed to the resolution of $A$ calculated. However, consideration of spectral data 10] shows that above the vibrational state $v=30$ an electronically excited state ${ }^{2} \Sigma^{+}$can be excited by a radiationless transition from the ground electronic state. Furthermore NO has an ionization energy $9.26 \mathrm{eV}$ somewhat greater than the dissociation energy, $6.5 \mathrm{eV}$. Therefore under strong fields the molecule might be excited electronically and suffer ionization before dissociation.

In summary, the following conclusions are found in comparison with single-mode driven lasers in previous work. Firstly, up to four thresholds were found for twocolor laser cases instead of three thresholds for singlemode laser excitation. Secondly, multiple transitions were found for initial states as high as 32, instead of 20 under a single-mode laser. Both the first and second points support that two-color cases are more complicated than single-mode cases. Thirdly, the tail of the dissociation curve remains almost constant, perhaps because our computation cannot resolve that fine detail. Fourthly, for most initial states the power required is not much influenced by the presence of the higher harmonic laser, although some initial states with smaller dissociation threshold were found. This conclusion disagrees with results of Charron et al. Finally, our result is consistent with those of Charron et al that for the first excited state a phase difference $\phi=\pi$ results in the greatest dissociation rate, although rotational effects are not yet considered. Because of the computational cost, only limited data were calculated.

I thank Professor How-Sen Wong for his constant support and Dr. J.F. Ogilvie for discussions.

1 Charron E, Giusti-Suzor A and Mies F H 1993 Phys. Rev. Lett., 71, 692.

${ }^{2}$ Yang X, Kim E K and Wodtke A M 1990 J. Chem. Phys. 93, 4483.

3 Schiemann S, Kuhn A, Steuerwald S and Bergmann K 1993, Phys. Rev. Lett., 71, 3637.

4 Ting J J-L 1994 J. Phys. B 27, 1249.

${ }^{5}$ Daboul J and Nieto M M 1994 Phys. Lett. A, in press, hepth 9405154 .

${ }^{6}$ Dauxois T, Peyrard M and Bishop A R 1993 Phys. Rev. E 47, 684 .

7 Heather R and Metiu H 1987 J. Chem. Phys. 86, 5009.

8 Ting J J-L, Yuan J M, and Jiang T F 1992 Comp. Phys. Comm. 70, 417.

9 Schinke R 1993 Photodissociation Dynamics, Cambridge University Press, pp 371-374.

10 Huber K P and Herzberg G 1979 Molecular Spectra and Molecular Structure IV. Constants of Diatomic Molecules, Van Nostrand Reinhold Co.

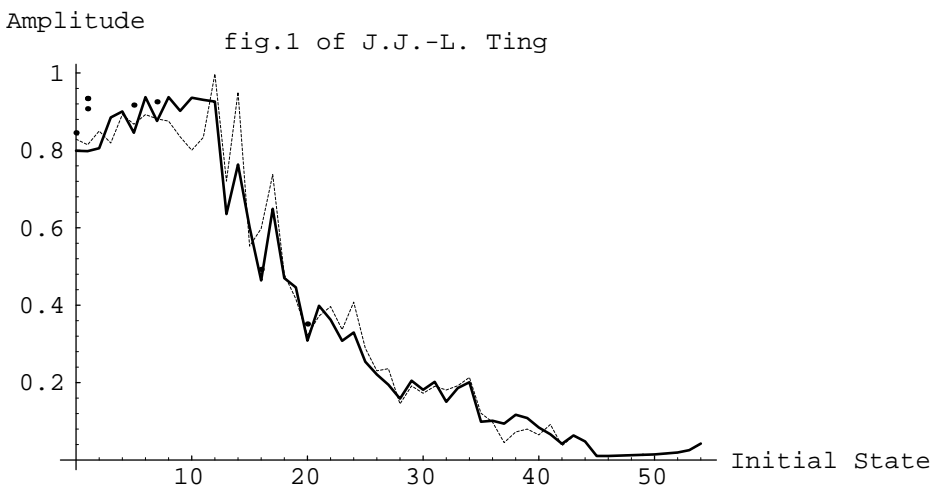

FIG. 1. Critical amplitude $\left(A_{c}\right)$ of various initial states with a single-mode laser at $\Omega=0.9$; for the solid thick line $T=300$ whereas for the thin dashed line $T=600$. 

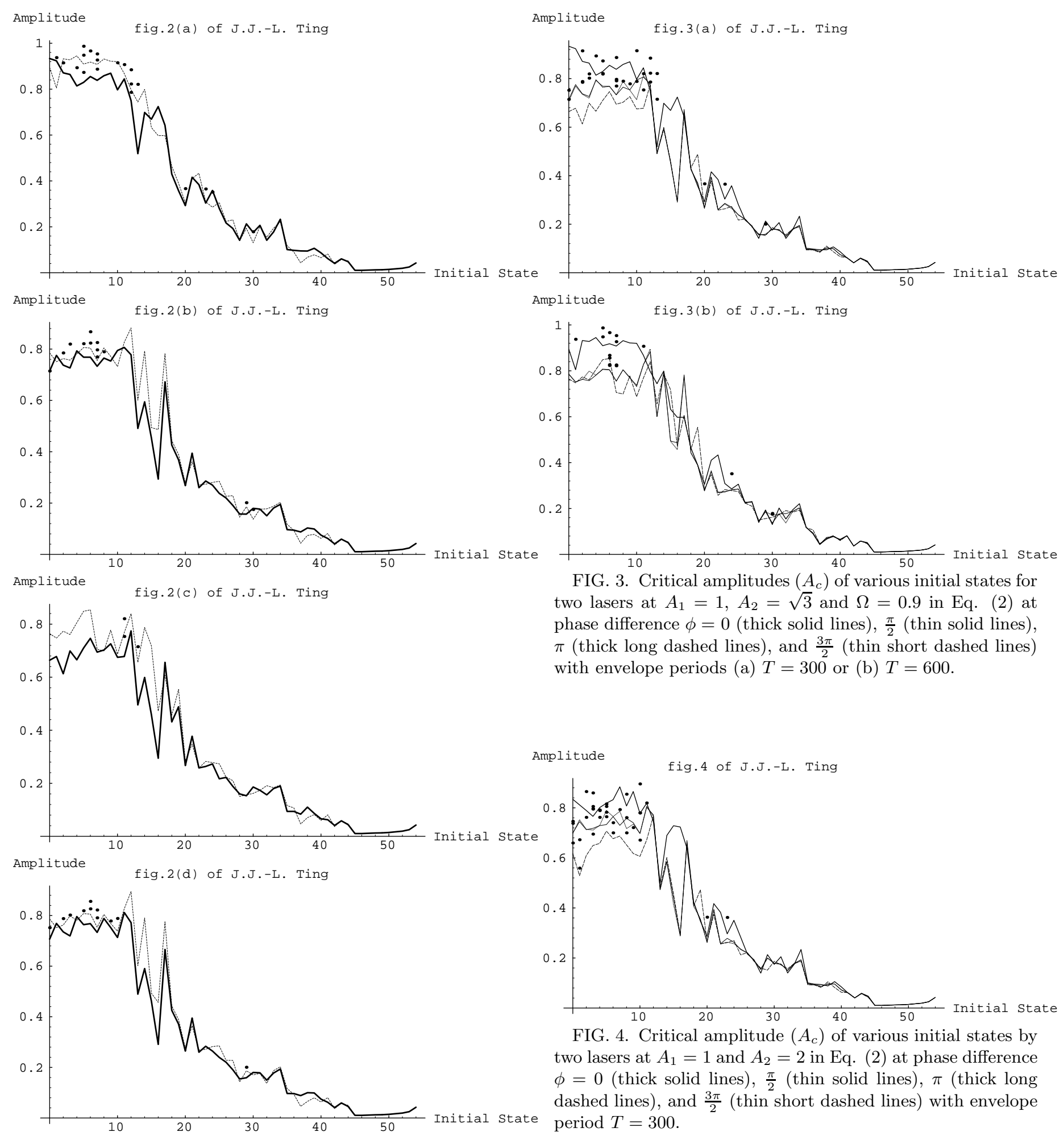

FIG. 3. Critical amplitudes $\left(A_{c}\right)$ of various initial states for two lasers at $A_{1}=1, A_{2}=\sqrt{3}$ and $\Omega=0.9$ in Eq. (2) at phase difference $\phi=0$ (thick solid lines), $\frac{\pi}{2}$ (thin solid lines), $\pi$ (thick long dashed lines), and $\frac{3 \pi}{2}$ (thin short dashed lines) with envelope periods (a) $T=300$ or (b) $T=600$.

FIG. 2. Critical amplitudes $\left(A_{c}\right)$ of various initial states with two lasers at $A_{1}=1, A_{2}=\sqrt{3}$ and $\Omega=0.9$ in Eq. (2) at phase difference $\phi=$ (a) 0 , (b) $\frac{\pi}{2}$, (c) $\pi$, (d) $\frac{3 \pi}{2}$ with $T=300$ (solid line) or $T=600$ (dashed line).

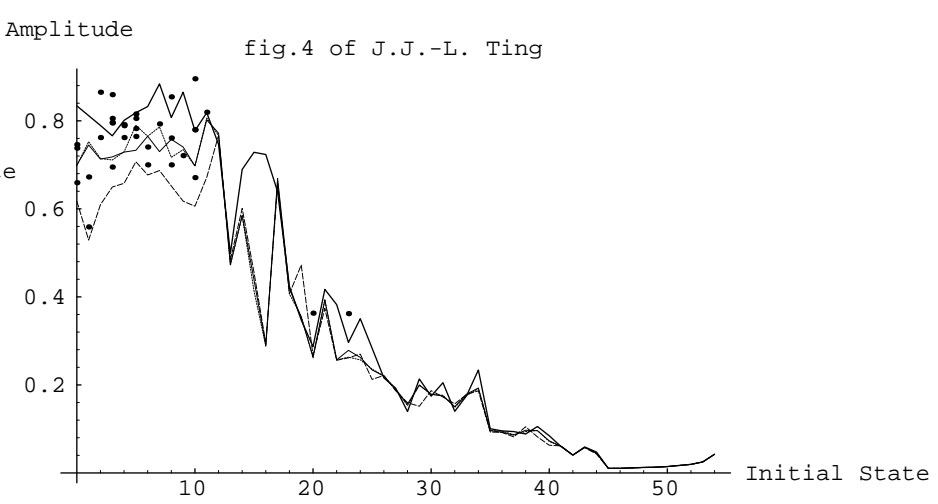

FIG. 4. Critical amplitude $\left(A_{c}\right)$ of various initial states by two lasers at $A_{1}=1$ and $A_{2}=2$ in Eq. (2) at phase difference $\phi=0$ (thick solid lines), $\frac{\pi}{2}$ (thin solid lines), $\pi$ (thick long dashed lines), and $\frac{3 \pi}{2}$ (thin short dashed lines) with envelope period $T=300$. 